\title{
A Consumer-Friendly Machine Learning Based Mechanism to Recognize the Quality of Commercially Available Fruits
}

\author{
Deepti C., Arjit Jindal, Prudhvi Reddy, Amrutha D.
}

\begin{abstract}
Cultivators and sellers of many high-in-demand fruits traditionally preferred natural ripening after picking. Greed of hefty profits has motivated some of them to artificially hasten the ripening process at the cost of people's health. Artificial ripening processes tend to degrade the entire quality of the fruit. The focus of this work is to describe a nondestructive method to detect artificial fruit ripening. To aid the detection, the proposed solution utilizes image processing and machine learning techniques to find the artificially ripened fruits. An input fruit image is selected as the test image. The next stage involves comparison of the features (histogram values) of the test image with the image of a naturally ripened one. A smartphone runs an android application to identify artificially ripened fruits. This work specifically concentrates on the commonly preferred Indian Mango and Indian Apple. The developed mechanism has an efficiency of $89-94 \%$ in correct detection.
\end{abstract}

Keywords: CNN based Approach, Fruit Ripening, Image Processing, Machine Learning.

\section{INTRODUCTION}

Fruit Ripening is a stage that makes the fruits to become suitable for consumption. The characteristics of ripe fruits are that they become sweeter, less green in color and acquire a softer texture. Ripe fruits show a marked increase in the acidic content. This increase in acidity content makes the fruit less acerbic. The acidic content can be measured by the Brix-Acid Ratio (BAR) [1]. BAR is a measure that considers the ratio of citrus product's Brix value to the anhydrous citric acid per 100 grams of the citrus product. The ripening process allows fruits to be described and classified as non-climacteric and climacteric. In climacteric fruits, the ripening process continues beyond harvesting. Indian climacteric fruits include banana, papaya, mango, guava, sapota, kiwi, fig, apple, passion fruit .They are expected to be consumed within a short time post ripening. The fruits that cannot be ripened

Revised Manuscript Received on February 05, 2020.

* Correspondence Author

Deepti C.*, PES University Electronic City Campus, Bengaluru, India.E-mail:deeptic@pes.edu. E-mail:arjitjindal87@hotmail.com

Prudhvi Reddy, PESIT Bangalore South Campus ,Bengaluru, India. E-mail:prudhvireddy.2310@gmail.com

Amrutha D, PESIT Bangalore South Campus, Bengaluru, India E-mail:amrutha12496@gmail.com.

(c) The Authors. Published by Blue Eyes Intelligence Engineering and Sciences Publication (BEIESP). This is an open access article under the CC BY-NC-ND license (http://creativecommons.org/licenses/by-nc-nd/4.0/)
Arjit Jindal, PESIT Bangalore South Campus, Bengaluru, India.

further after they are harvested are referred to as non-climacteric fruits. These fruits generate minute quantities of ethylene. A significant increase in the rate of respiration and carbon dioxide production is not observed. Orange, Mousambi, Grapes, Pomegranate, Litchi, Watermelon, and Cashew are some of the common non-climacteric Indian fruits.

Scarcity of quick, less complicated and uniform fruit ripening methods is a predominant problem during fruit production, harvesting and sale. Conventional ripening methods and modern chemical ripening methods have their own merits and demerits. The total days required for gradual ripening of edible fruits varies based on the existing climatic condition and the variety of fruit. For example, it takes approximately five to six days for ripening of mangoes and six to seven days for ripening of sapota. This has led to a rise in the employment of illegal methods illegally employed to quickly ripen fruits. One such mechanism is the use of ethylene liquid that can make mangoes ripen in a day or two thus making them fit for consumption. Concerns with regard to public health have brought forth a need for a mechanism to determine whether the fruit is artificially ripened or naturally ripened.

\section{LITERATURE SURVEY}

Evaluation of the quality of fruits after harvesting using destructive or non-destructive methods is a common approach. Destructive methods damage the fruits physically, it is time consuming and hence it is not an optimal approach. Sophisticated laboratory measurements of total soluble solids (TSS) [1], sugars, acids, pigments and nutrients carried out using traditional instruments such as refractometer and spectrophotometer are usually inaccessible to common people along with the additional disadvantage of being highly expensive. The study by Camargo [2] labels the visual symptoms of plant diseases through an analysis of coloured images. The focus of this work is directed towards on-plant assessment of fruit ripening which is cumbersome. The study by Ketki and Bavaskar [3] recommended an image recognition based scheme to recognize the status of ripening on fruits that were still on the tree. For counting the fruits, the main issue was inconstant illumination conditions in the outdoor environment. An added problem was the obstruction of capture of the ideal fruit image [4] by leaves, branches, and other objects. A study conducted by Mohammad Asif in 2012 [5] discusses the use of substandard substances like calcium carbide in order to fasten the ripening of fruits for domestic markets. 
It also shows its toxic effects on human health. The work by Mustafa and Khairul [6] is centered on the stage wise development of mangoes.

The developed image processing algorithm was run on Raspberry Pi .It applied K-means clustering to decide the fruit colour and size.

The highlight of the research work by Srividhya, Sujatha and Ponmagal [7] was that it could sense the presence of ethylene gas by using soft sensors that were built on the basis of Image Processing and Artificial Neural Networks (ANN) algorithms. The drawback of this technique is that it relied on the colour of the fruit at various stages in ripening. The work by Karthika, Ragadevi and Asvini [8] proposed an image processing technique that examined statistical features in order to segregate artificially ripened bananas using support vector machine classifier.

Visual scrutiny has been found to be insufficient to conclude whether the fruit under observation has matured naturally or not. To aid the detection Maheswaran and team [9] have developed a device which uses image processing to find the artificially ripened mangoes. The system has an efficiency of $91 \%$ in the identification of the fruits ripened artificially. This survey concludes that the existing systems utilize sensors and laboratory equipment. Analysis is made based on the chemical composition of the fruit and total soluble solids which could physically damage the fruit. It is also a timing consuming process. The limitations of the existing systems allow a new system to be considered for quick and efficient identification of ripened fruit using CNN.

\section{SYSTEM REQUIREMENTS}

\section{A. Functional Requirements}

The system should provide the user the option to capture the image of the specimen i.e. fruit. Image captured by the System should be given to the application as the input.

The image should be processed to check the image has a fruit or not. The input image should be processed to isolate only the fruit boundaries. The application should use the processed image to get the desired output. System should also guide the user about the availability of naturally ripened fruits in organic stores.

\section{B. Non Functional Requirements}

An approximate accuracy of around 90-95\% of accuracy in fruit classification is expected.. The System has to be reliable in identifying the fruit whether it is artificial or naturally ripened fruit based on number of instances. The expectation is to achieve 80-90 correct identifications among 100 instances approximately. The system needs to be scalable to fruits those can be classified based on external color features.

\section{Assumptions and Dependencies}

The orientation of the fruit under consideration is appropriate The time lag between capturing the picture and obtaining the result is negligible. It is sensitive to lighting conditions. The distance from which the image is captured is far/near enough for the detection process.

\section{Constraints}

Any other object other than fruits should not be accepted by the application. Appropriate distance and orientation of the fruits is required. Only one image is processed at a time. A proper lighting condition is necessary.

\section{SYSTEM ARCHITECTURE}

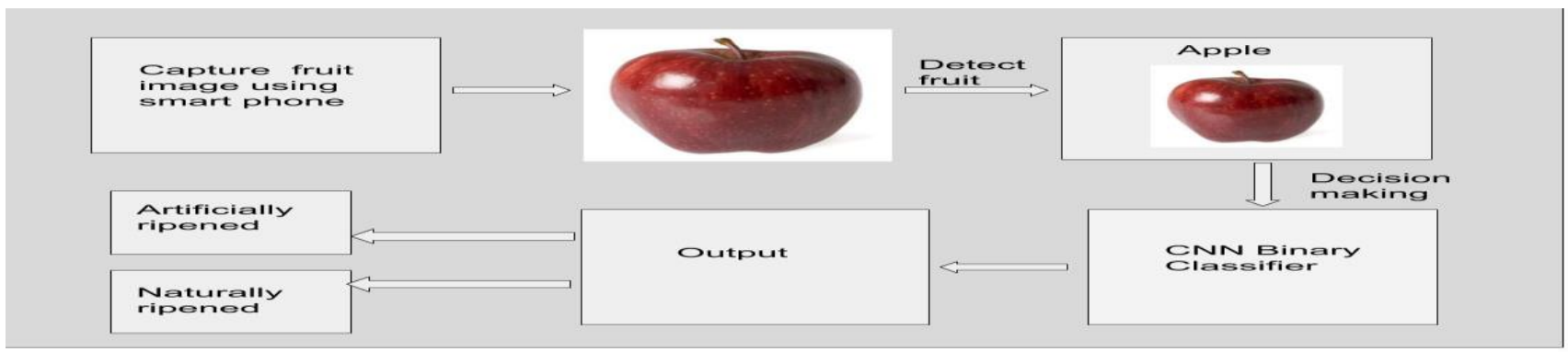

Fig. 1 Overview of the System Architecture

Fig. 1 gives an impression of the complete system. The process takes place in 3 stages:

1) The image is given as input

2) The next stage is processing, in which it is identified whether a fruit is there in the image or not.
3) The class label assigned as whether it is fruit or not. Finally, the result is passed to the next level of the architecture to classify the fruit as artificially ripened or naturally ripened. 


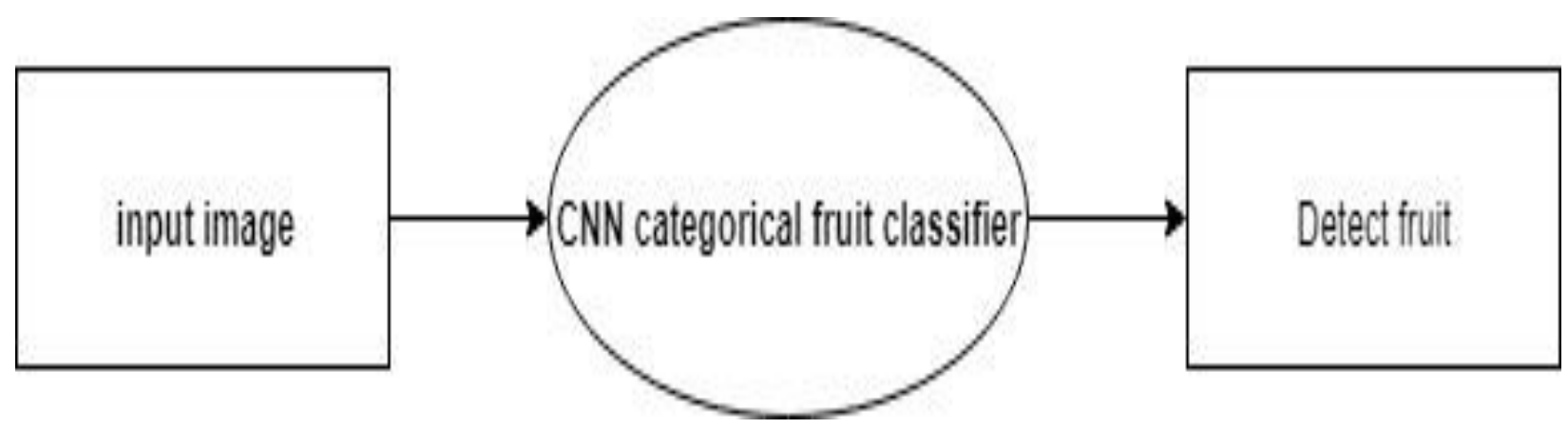

Fig. 2 Classification Process

Fig. 2 gives an overview of the classification system. The process takes place in 4 stages:

a) The image is taken as input

b) The next stage is processing, where the fruits are classified using CNN. c) The class label assigned is whether it is artificial or natural.

d) Finally, the result is displayed on the smart phone based on the decision made in the processing stage

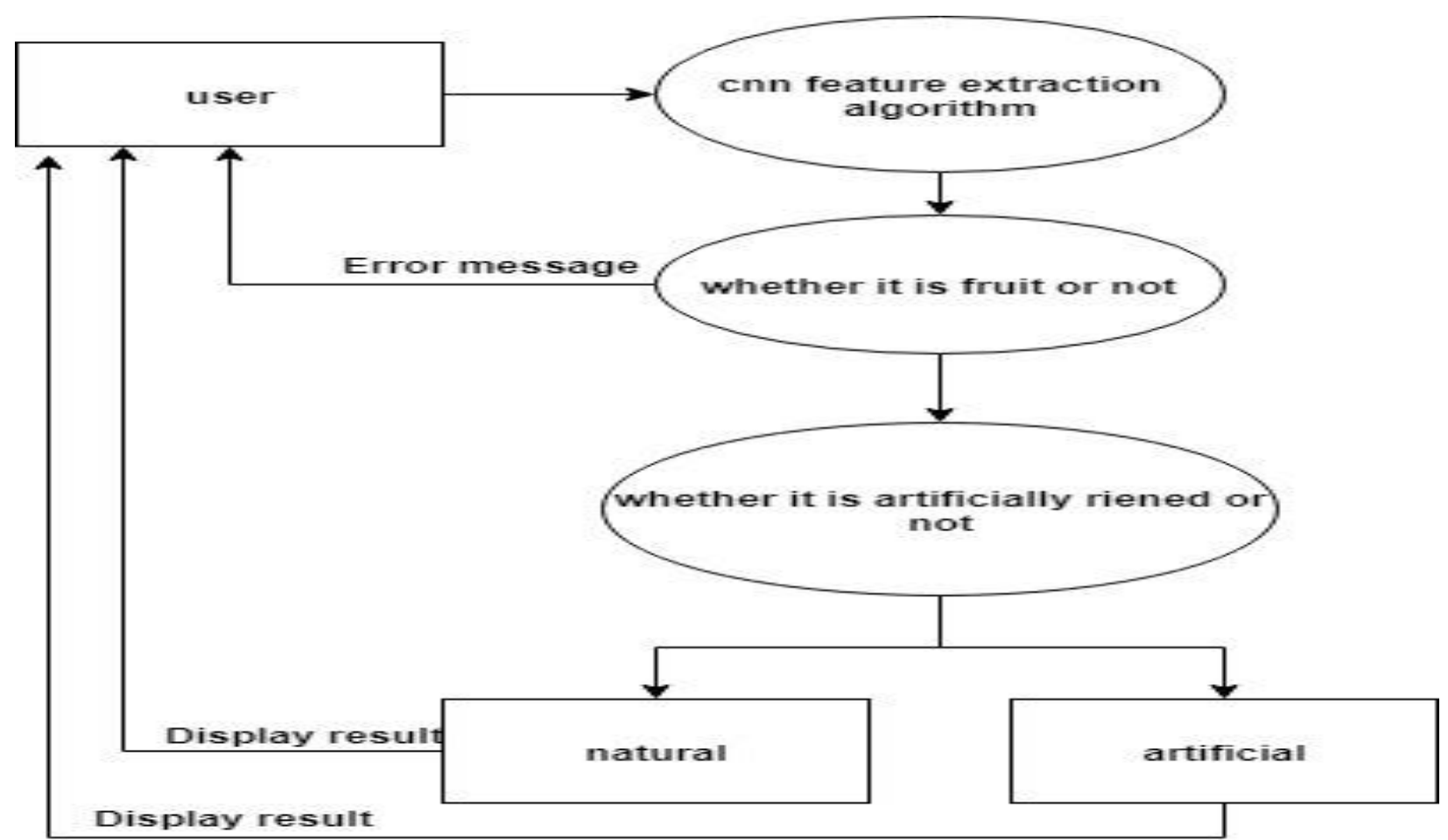

Fig. 3 Implementation Flow

Fig. 3 shows the 5 stages involved in implementation

1) User captures the image of the fruit as input

2) $\mathrm{CNN}$ is used to find whether the fruit is artificial or natural

3) Error message is displayed to the user indicating that the image is not a fruit

4) Results of the classification are displayed. 


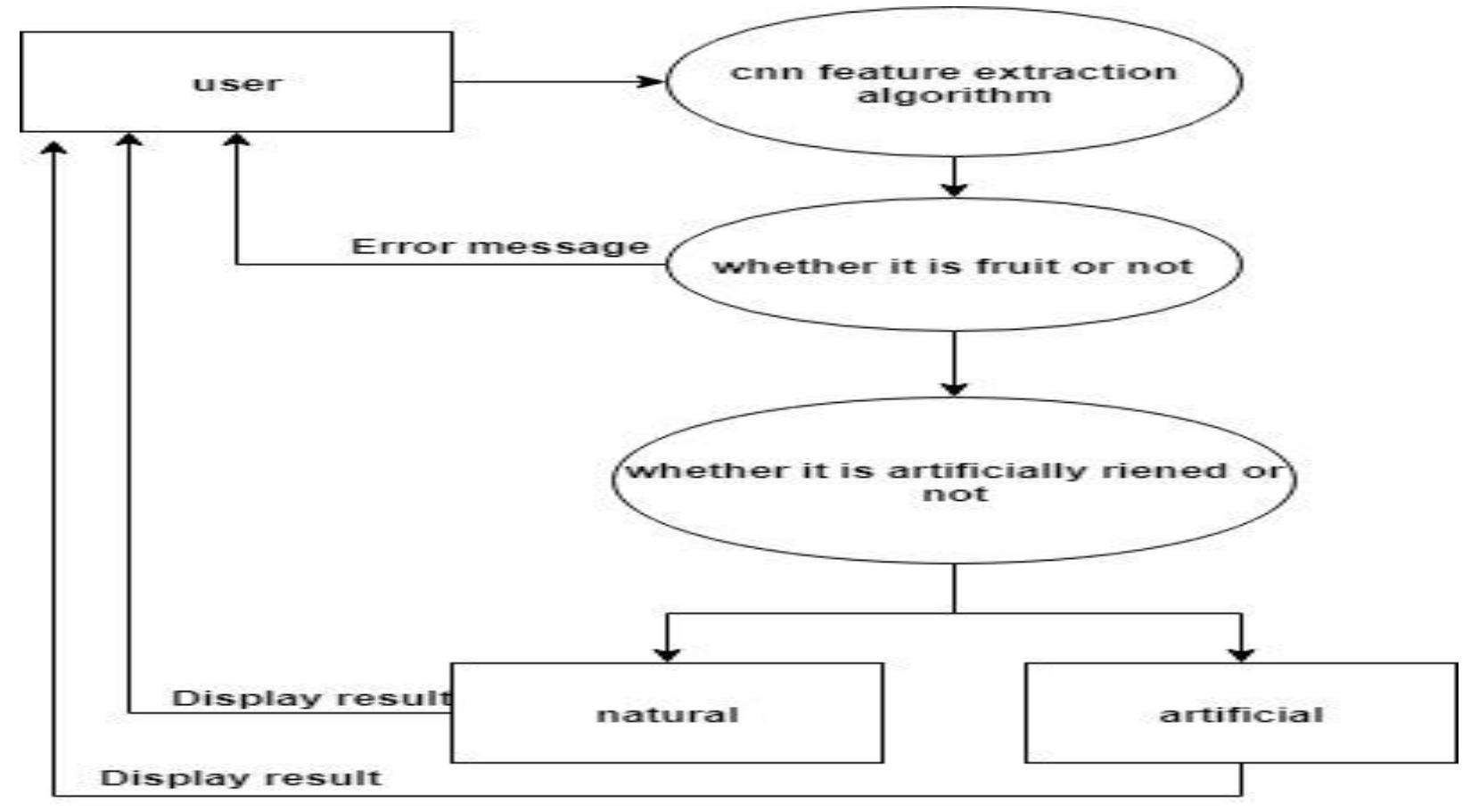

Fig. 4 Error handling Process

Fig. 4 elaborates on the error handling mechanism.

When the brightness of the image is below the required brightness of image processing, an error report is sent to the user.

1) When the image is not a fruit, the error message is displayed to the user.
2) If the brightness is normal and the image is a fruit, only then the fruit is classified using the binary CNN.

3) The result is displayed.

4) The user is allowed to provide feedback

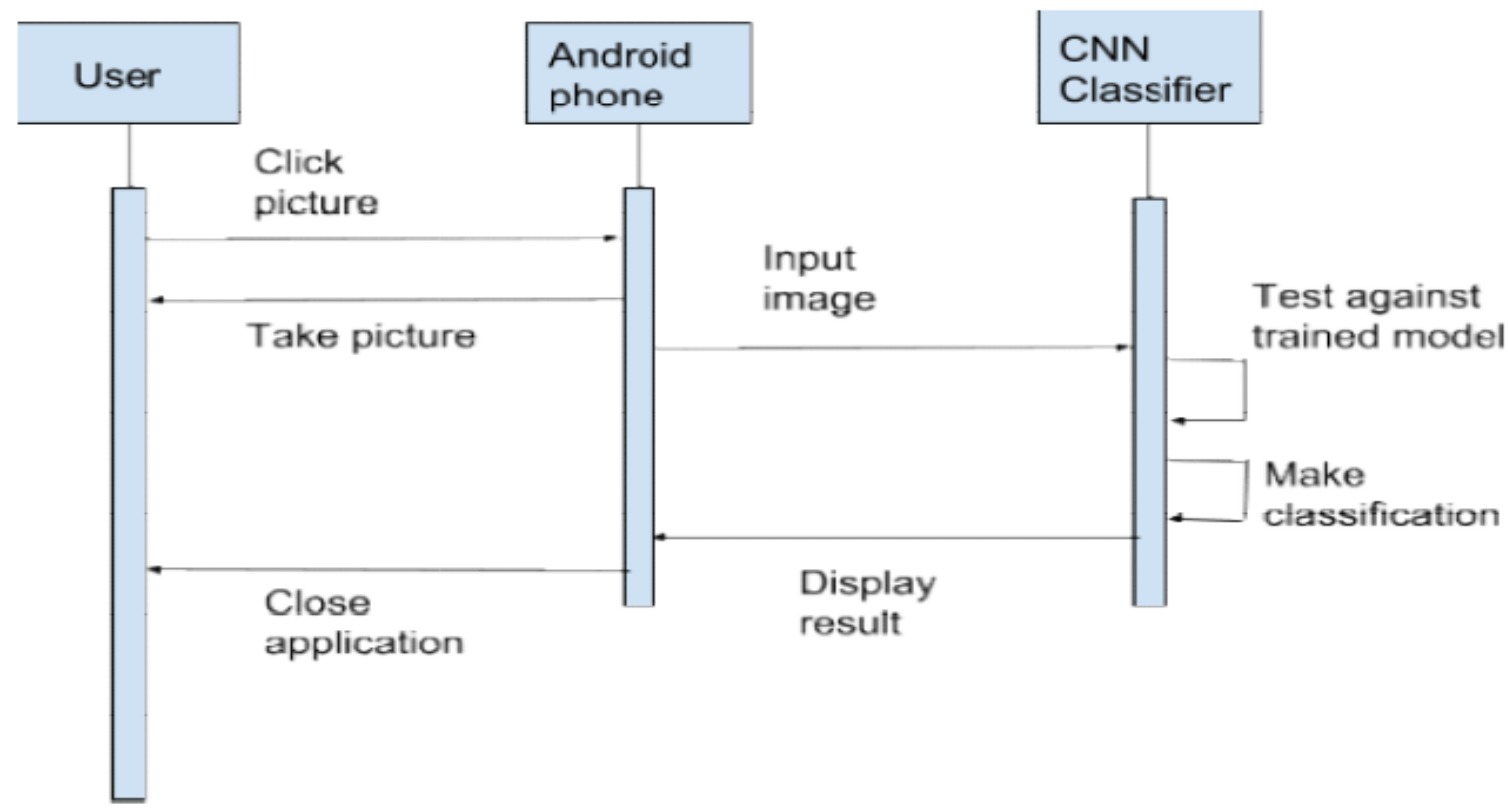

Fig. 5 Sequence Diagram

Fig. 5 illustrates the interaction between the elements on the parallel axis. The objects shown are the user, Android phone, CNN Classifier. The vertical axis represents time proceedings.
1. User clicks the picture on smart phone and then sends it to the classifier in the sequence.

2. The classifier tests the image, then classifies the image and then sends it to the phone display.

3. User can see the result and closes the application 


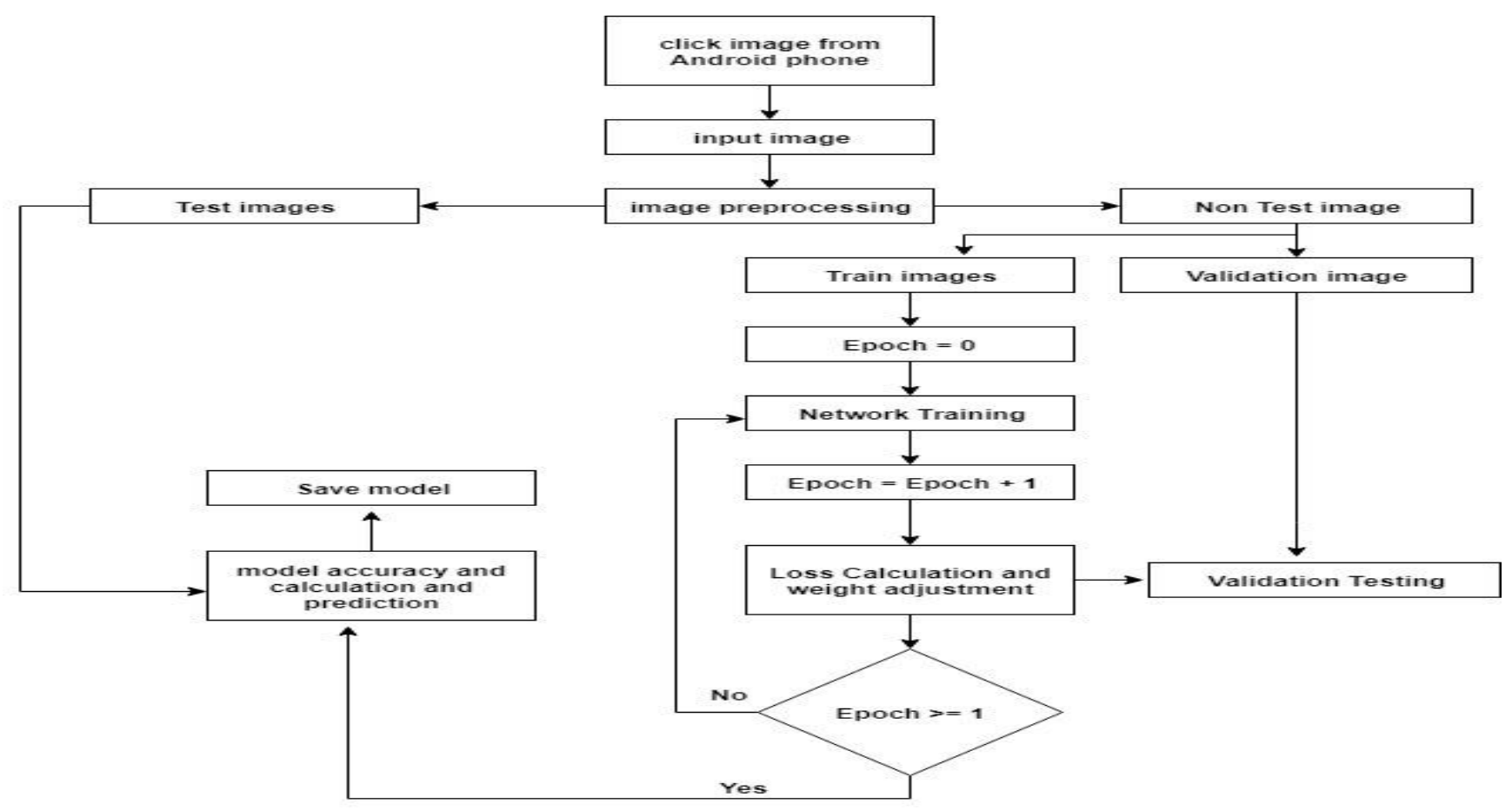

Fig. 6 Training and Classification

Fig. 6 explains the training and classification procedure.

1) The training images are used to direct the model to learn the features of the images and adjust the weights.
2) Each epoch will train the model with the images in the datasets. Loss calculation is done with the validation dataset and weights are adjusted accordingly

3) Increase the number of epochs until the accuracy is reached and the model is saved

4) This model is used for testing the images that are taken with the smart phone

\section{RESULTS AND SNAPSHOTS}

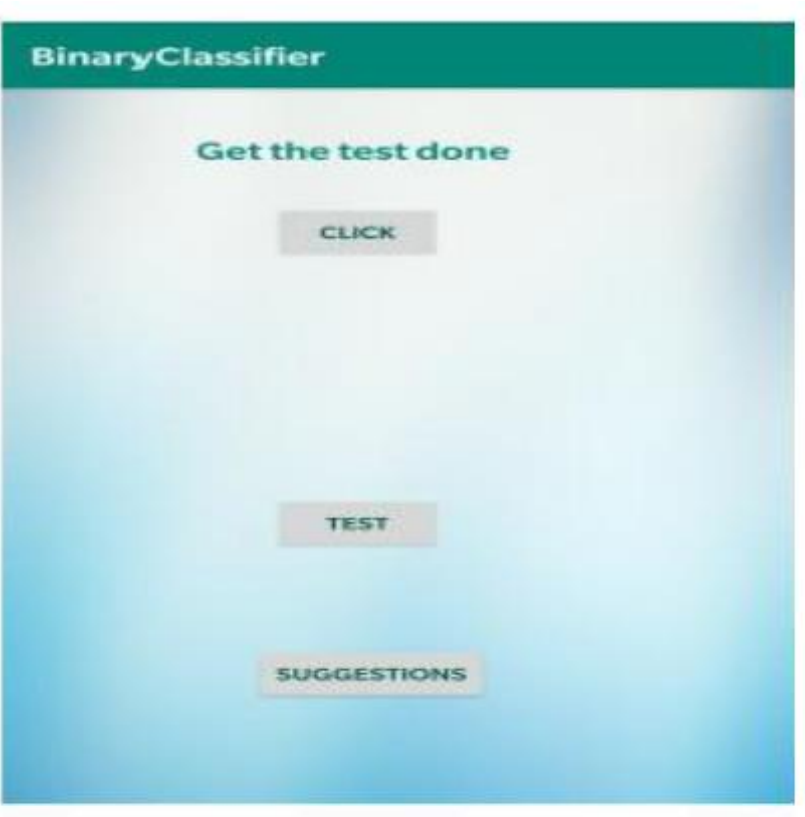

Fig. 7 Welcome Screen

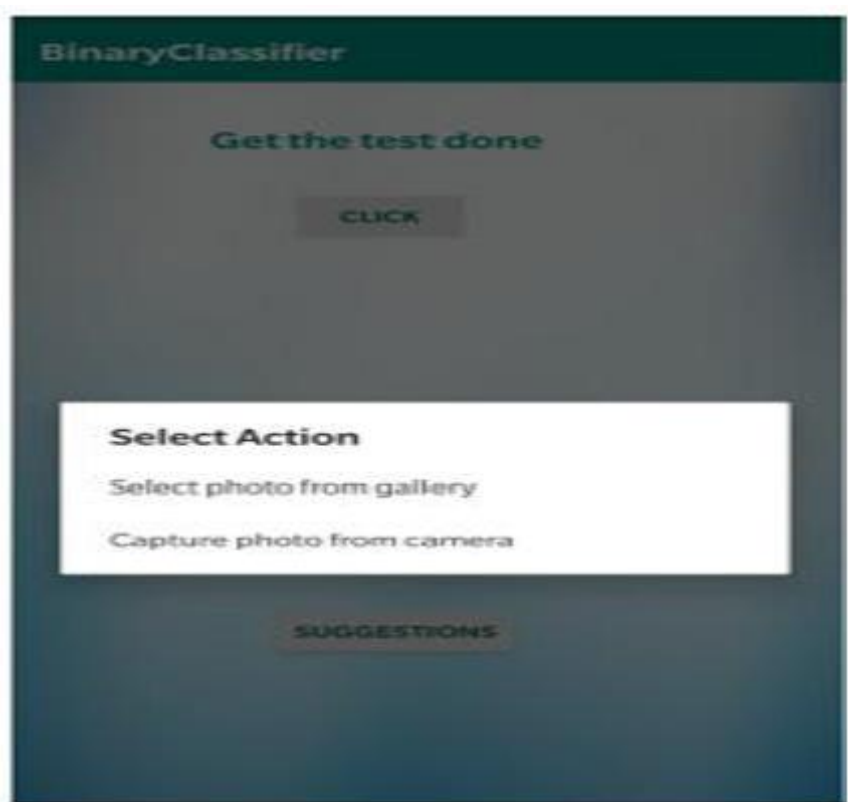

Fig. 8 Input Image 
A Consumer-Friendly Machine Learning Based Mechanism to Recognize the Quality of Commercially Available Fruits

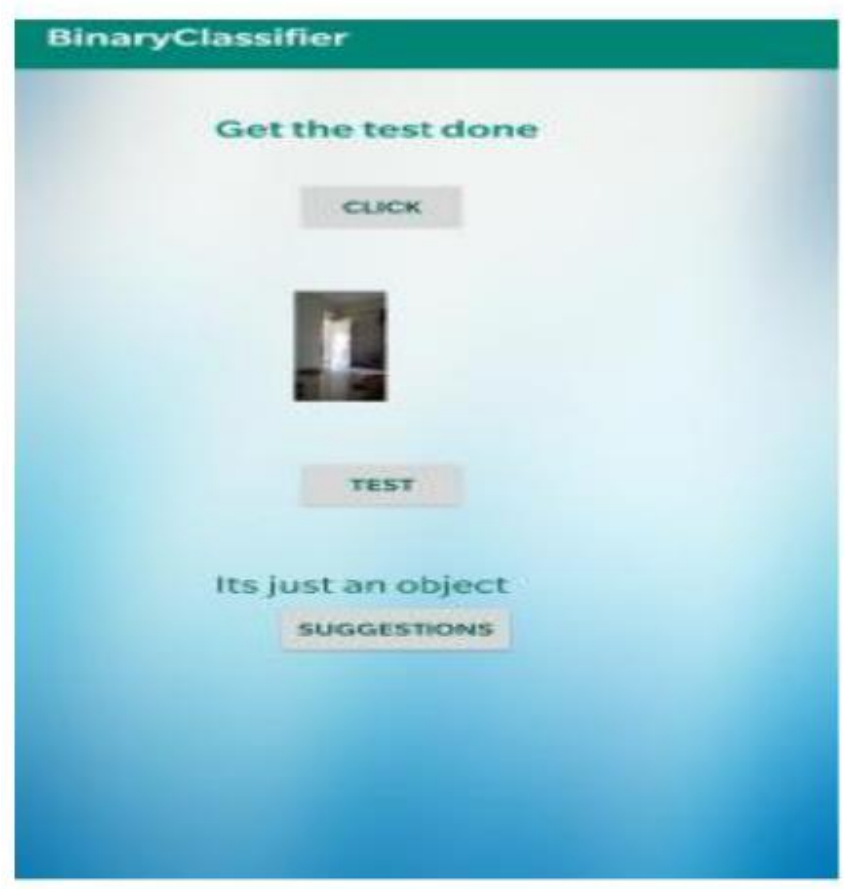

Fig. 9 Fruit not Detected in Input Image

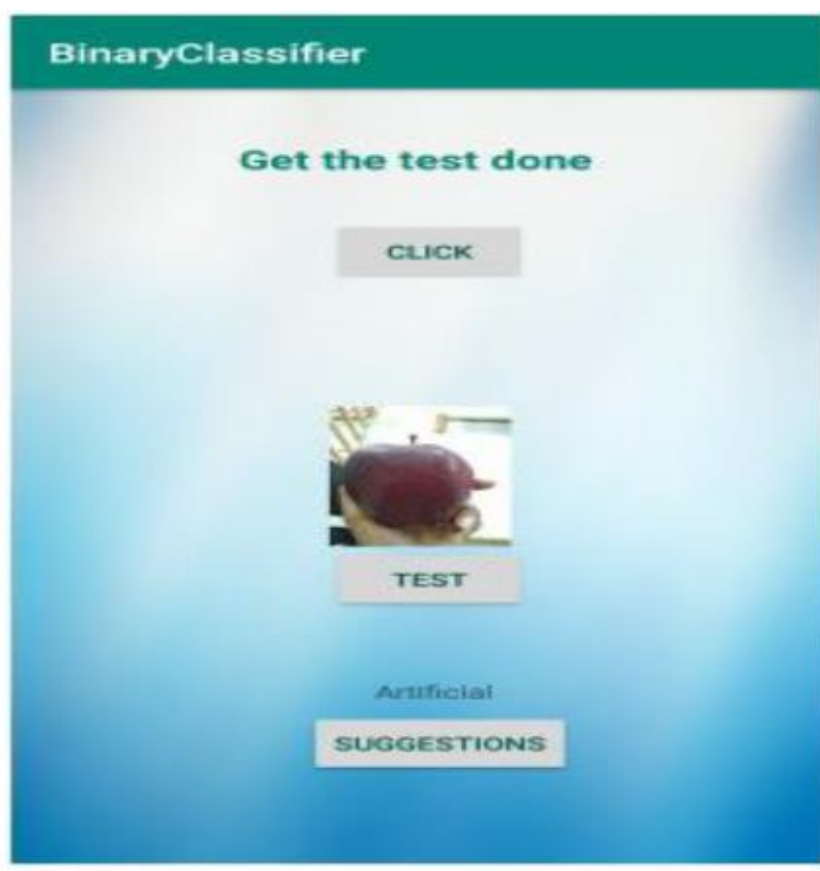

Fig. 10 Fruit Classified as Artificial

detect artificial fruit ripening which has more significant

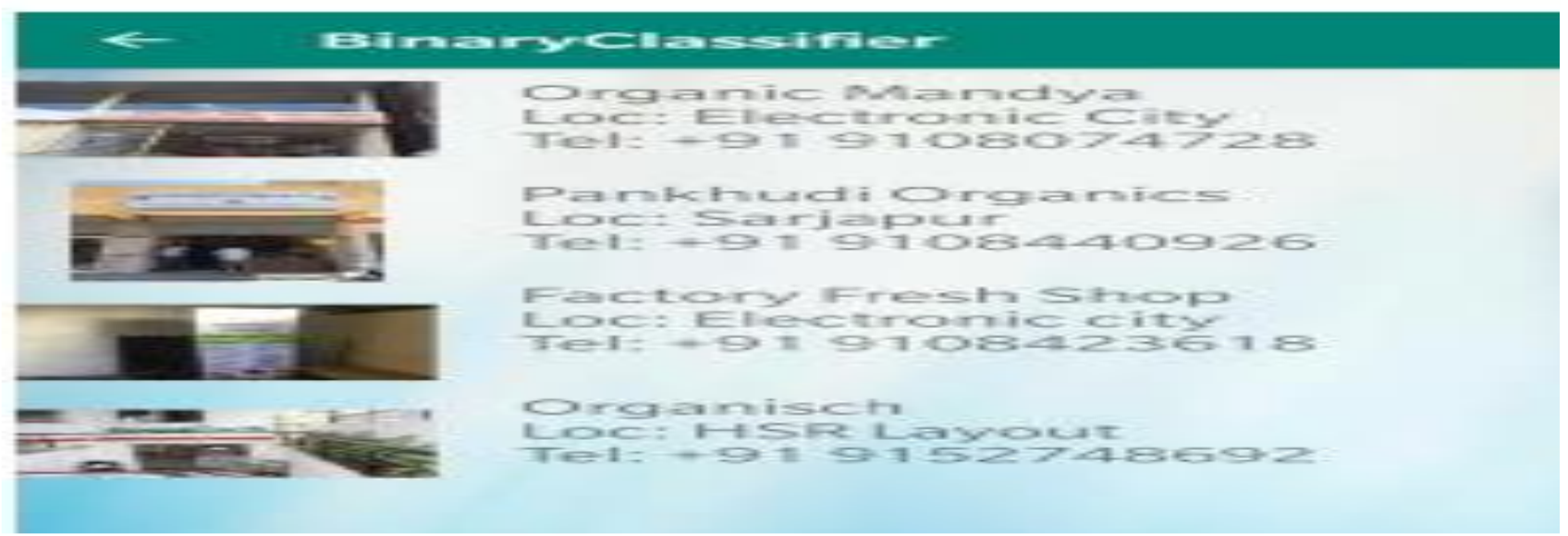

Fig. 11 Nearby Organic Shops Indicated

advantages compared to destructive methods. Image

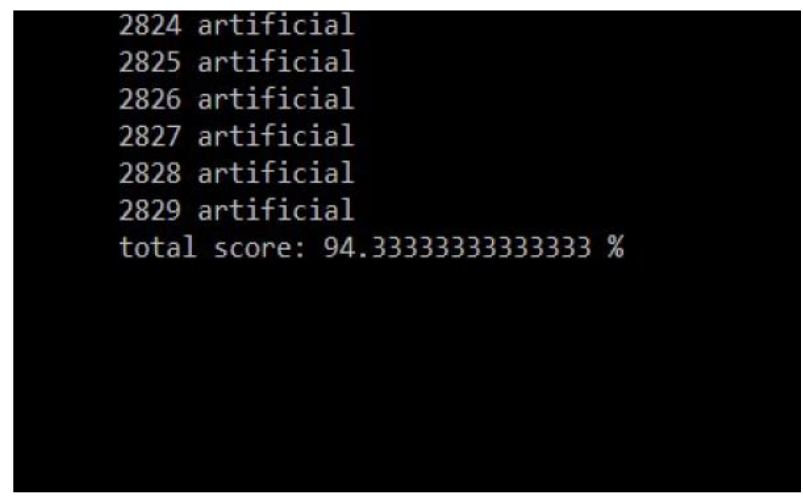

Fig. 12 Efficiency of the Model for Mango

\section{CONCLUSION AND FUTURE WORK}

The idea of this paper is to explore the most efficient and cost effective way in which artificially ripened fruits can be detected. The focus is to develop a non-destructive method to

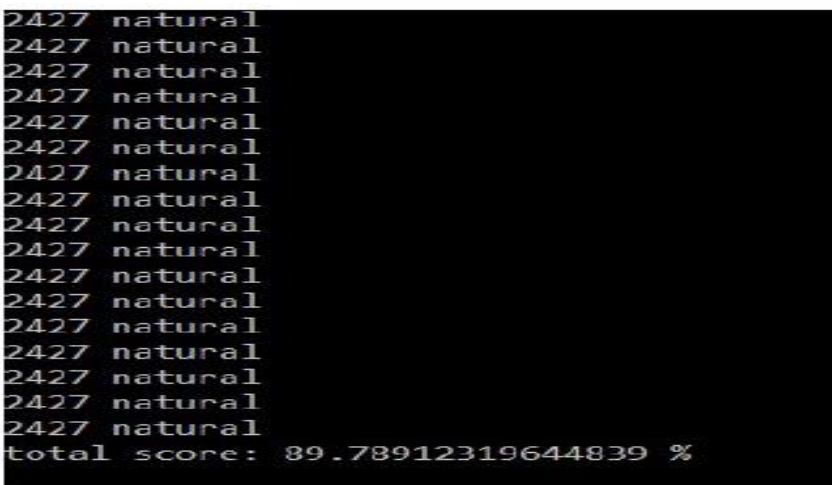

Fig. 13 Efficiency of the Model for Apple

processing methods are utilized to identify the unnaturally matured fruits without causing physical damage to the fruit.

Blue Eyes Intelligence Engineering \& Sciences Publication

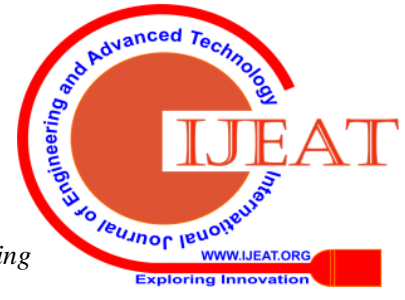


The main objective is to provide general consumers with the ability to detect whether the fruit is naturally or artificially ripened by just scanning the fruit using smart phone. The result is at his or her fingertips.

The system can be improved by making it available for more fruits and vegetables for a substantial increase in usability and scalability. The user interface of the mobile application can be improved to make it more user friendly and more aesthetic in appearance.

\section{REFERENCES}

1. Magwaza, Lembe Samukelo, and Umezuruike Linus Opara. "Analytical methods for determination of sugars and sweetness of horticultural products—A review." Scientia Horticulturae 184 (2015): 179-192.

2. Camargo \& J S Smith. "An Image-processing Based Algorithm to Automatically Identify Plant Disease Visual Symptoms", Biosystems Engineering, Issue No.1, Pages 9-21, 2009.

3. Ketki Tarale, Anil Bavaskar, "Fruit Detection Using Morphological Image Processing Technique", ICSESD, 2017.

4. Y. Çakır, M. Kırcı, E. O. Güneş and B. B. Üstündağ, "Detection of oranges in outdoor conditions," 2013 Second International Conference on Agro-Geoinformatics (Agro-Geoinformatics), Fairfax, VA, 2013, pp. 500-503.

5. Asif Mohammad,"Physico-chemical properties and toxic effect of fruit ripening agent Calcium Carbide ",Annals of tropical medicine and public health, Volume 5,Issue No.3 ,Pages 150,2012.

6. Izadora Binti Mustaffa, Syawal Fikri Bin Mohd Khairul. "Identification of Fruit Size and Maturity Through Fruit Images Using OpenCV-Python and Raspberry Pi", ICORAS, 2017.

7. V. Srividhya, K. Sujatha "Ethylene Gas Measurement for Ripening of Fruits Using Image Processing", Indian Journal of Science and Technology, 2016.

8. R. Karthika, K.V.M.Ragadevi and N.Asvani. "Detection of artificially ripened fruits using Image processing", International Journal of Advanced Science and Engineering Research 2017.

9. Dr.S.Maheswaran, S.Sathesh. "Identification of Artificially Ripened Fruits Using Smart Phones", International Conference on Intelligent Computing and Control, 2017.

\section{AUTHORS PROFILE}

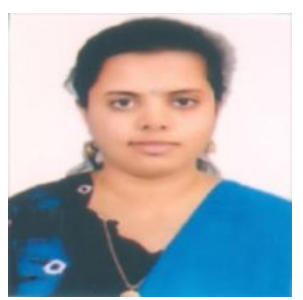

Deepti C currently holds a faculty position as Assistant Professor, Department of CSE, PES University Electronic City Campus. She received her Bachelor of Engineering degree in Electronics and Communication in 2004. She received her M.Tech in Computer Network Engineering with distinction from Visvesvaraya Technological University in 2009..She is pursuing research currently in the area of Internet of Things. Her main research interests are Internet of Things (IOT), wireless sensor networks and wireless network security.

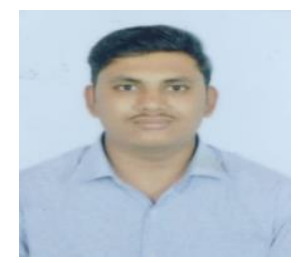

Prudhvi Kumar Reddy is currently pursuing his masters in computer science at University of Alabama Birmingham, USA. He completed his graduation in Information Science and Engineering at PESIT Bangalore South Campus in June 2019.During the course of his Bachelor's degree he has worked on a project named "Identification of Artificially Ripened Fruits Using Smart Phones", He contributed extensively towards preparing the dataset and backend work of the project.

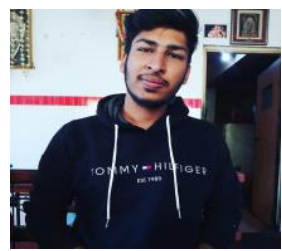

Arjit Jindal is currently at UNACADEMY with a goal in improving customer experience. He is passionate about coding .He gets his dopamine by working on the push from code to production. He completed his graduation in Information Science and Engineering at PESIT Bangalore South Campus in June 2019.During the course of his Bachelor's degree he has worked on a project named "Identification of Artificially Ripened Fruits Using Smart Phones".

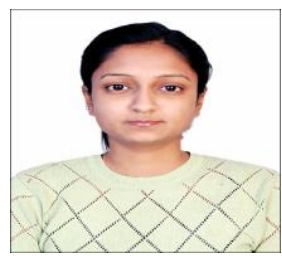

Amrutha D is currently undergoing training for her role as a Data Scientist at TEKION INDIA PRIVATE LIMITED. She has completed her graduation in Information Science and Engineering at PESIT Bangalore South Campus in June 2019.During the course of her Bachelor's degree she has worked on a project named "Identification of Artificially Ripened Fruits Using Smart Phones". 